\title{
Convergence Test of Model Reference Parameter Adaptive SRM Drives
}

\author{
László Számel \\ BUDAPEST UNIVERSITY OF TECHNOLOGY AND ECONOMICS \\ H-1111 Budapest, Egry József u. 18. \\ Budapest, Hungary \\ Tel.: $+36 /(1)-463.29 .71$. \\ Fax: $+36 /(1)-463.36 .00$. \\ E-Mail: szamel@eik.bme.hu \\ URL: http://www.vgt.bme.hu/info/cv/szamellaszlo.htm
}

\section{Acknowledgements}

This paper was supported by the Hungarian National Scientific Funds (OTKA Ref. No.T042866), for which the author expresses his sincere gratitude.

\section{Keywords}

«Adaptive Control», «Robust control», «Servo-drive», «Simulation», «Switched reluctance drive»

\begin{abstract}
Nowadays switched reluctance motor (SRM) drives have been widely used in the field of controlled electric motor drives. The paper proposes a model reference parameter adaptive control method for SRM drives. The main goal of the drive control is to improve dynamical performance by compensating for the motor nonlinearities. The adaptation practically works only in a relatively narrow speed error track (adaptation range) which is equal to approximately 20-100 revolution/min absolute value of speed error. The drawback of this limitation is the relatively short time for the algorithm to operate. But the convergence of the algorithm is extremely fast which significantly reduces the effect of this drawback. Two more important advantages emerge when adaptation works only with small speed errors. First of all the controller at changing drive parameters adapts to parameters around the value specified by speed reference signal which also assists to speed the adaptation. The other significant positive effect is the disappearing of the problem coming from nonlinear systems that the response of the system can even differ in its character when the value, amplitude of the reference signal is changed. The convergence test of this control method was performed in an experimental drive system. A SRM of $6 / 8$ pole and $4 \mathrm{~kW}$ rated power was used. Simulation and experimental results are presented.
\end{abstract}

\section{Introduction}

In motion control systems are robustness against parameter changes and disturbance rejection of main interest. The model reference adaptive control has the following features:

- It makes the compliance of the system with varying operational conditions possible and ensures the behavior of the controlled system according to the prescribed reference model.

- It means such a special type of adaptive systems which results in nonlinear control systems. This is the reason why the analytical analysis is completed by Lyapunov stability criterium or by hyper-stability principle.

- Its planning and application is closely related to the using of computer methods.

- Simple realisation of the control algorithm.

In this paper the application of a model reference parameter adaptive control to switched reluctance motors is presented. 


\section{Drive system}

The block scheme of the examined drive system is shown in Fig.1. The supply unit consists of three main blocks, namely the RECTIFIER, the FILTER and the INVERTER. The inverter is a pulsed width modulated (PWM) one, marked with QP in the figure and it contains one-one switching transistor per phase and a brake chopper, not shown in the figure. The common point of phase windings is supplied by the PWM inverter. It is of autonomous operation and has an inner current control loop. The other ends of phase windings are connected to the phase switching transistors.

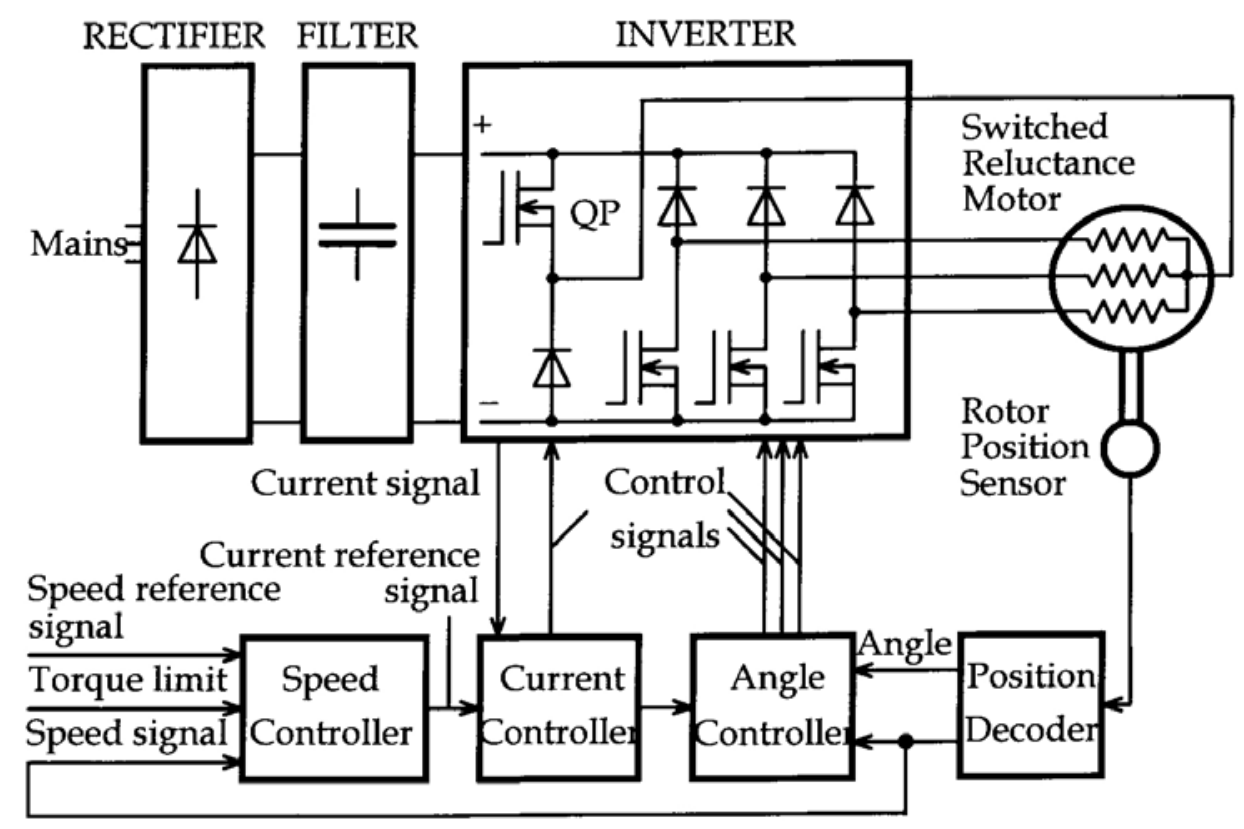

Fig. 1: Block scheme of drive system

The Position Decoder has two outputs: the Angle and Speed signals. Based on the two signals, the Angle Controller composes the Control signals for the phase switching transistors.

Fundamentally, SRM drives have two control loops [3], the outer one is the speed loop, Speed Controller and the inner one is the current loop, Current Controller. The output signal of the Speed Controller serves for a Current reference signal of the Current Controller. The hardware and software tools together fulfil the two-loop control. The Current Controller produces the control signal for the PWM inverter, and receives the Current signal from the PWM inverter at the same time.

\section{Control of the SRM drive}

\section{Current control}

The Current Controller is totally based on its hardware solution. Based on the current reference signal, it controls the PWM inverter of fix frequency by installing an analog controller. The current feedback also comes from the PWM inverter. For the control of the sum of phase currents (Fig. 1.) it is suitable a simpler four-transistor inverter [1] and is not necessary a six-transistor one as in the case of control of phase currents independently from each other. But the detriment of the previous solution is that the torque pulsation can be decreased in a smaller degree by changing the turn-on and turn-off angles. 
Namely, in the case of the constant current reference signal the current increase is limited by the switchedoff, but conducting phase current as the regulator controls the sum of two phase currents. The increase of the phase current at starting the conducting state can be forced by the modification of the current reference signal [6]:

$$
i_{r}=u \sum_{j=1}^{3} C_{j}+\sum_{j=1}^{3}\left(1-C_{j}\right) \cdot i_{j},
$$

where:

$i_{r} \quad$ is the current reference signal,

$i_{j} \quad$ is the current signal of phase $j$,

$u \quad$ is the output of the speed controller,

$C_{j} \quad$ is the control signal of phase $j$ (0 or 1$)$.

The supplement of the first member of Eq. 1 makes the overlap of the phase conduction possible, while the effect of second member is to increase the reference signal with the current of the switched-off, but not current-free phase.

The ripple free operation can be realize only with a current waveform depending on the angle, speed and torque [5]. The proposed ripple reduced method [6] changes only the turn-on and the turn-off angle in function of the speed and current reference. The optimum turn-on and turn-off angles of the SRM drive has been determined by computer simulation based on the measured results of the analysed drive.

The angle control of the drive determines the actual turn-on and turn-off angles with a two-variable interpolation from the results stored in a look-up table and calculated by the above method.

\section{Speed control}

A model reference parameter adaptive control is used for the speed control. Such an adaptive control has been succesfully elaborated by using a suitable chosen Lyapunov function to compensate the gain of the speed control loop [4] [7] [8] [9].

The adaptive control of servo-drives with cascade arrangement is most effective if it is applied in the inner loop containing the effect of variable parameters directly, i.e. the inertia $\left(J_{m}\right)$ and/or torque factor $\left(k_{m}\right)$. The speed control implemented with PI controller is of cascade arrangement in fact as it contains an inner, proportional feedback loop (PF controller, [2], Fig. 2.). A one-storage proportional element can describe this inner loop neglecting the time constant of the closed current control loop. By this our adaptation algorithm will be simplest.

The relation between the accelerating current determining the dynamic torque and angular velocity can be given by the following transfer function:

$$
Y_{\omega,\left(i-i_{t}\right)}(s)=\frac{A_{i}}{s}
$$

where $A_{i}=\frac{k_{m}}{J_{m}}$.

The arrangement of control circuit can be seen in Fig. 2. The section determined by the transfer function $Y_{\omega,\left(i-i_{t}\right)}(s)$ is fed back by a proportional member of gain $K_{P}$. The task is to change the gain $K_{P}$ in such a way that the product $A_{i} K_{P}$ should remain constant despite the change of parameter $A_{i}$. The transfer 
factor of the inner closed loop is given by the reciprocal $\left(1 / K_{P}\right)$ of the feedback member that is not constant because of the torque factor change. In the consequence of above the loop gain of the outer speed

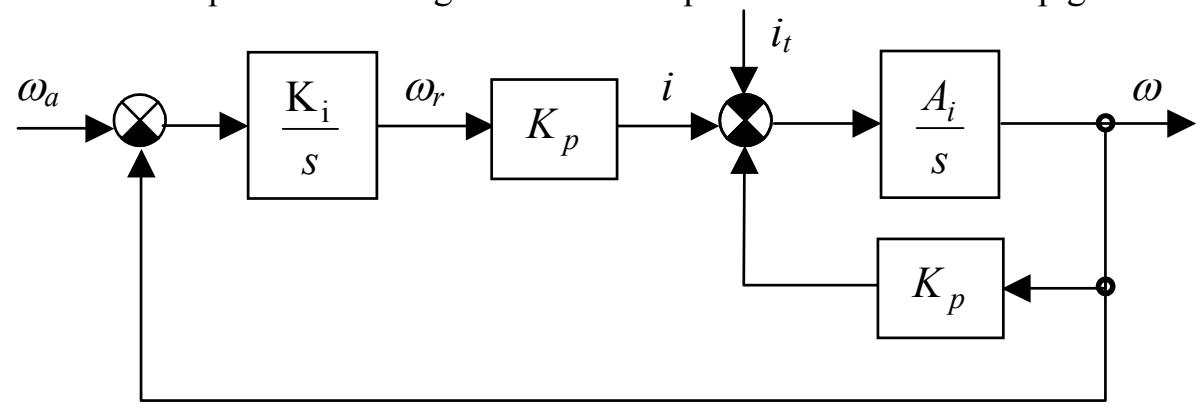

Fig. 2: Block scheme of speed control

control loop would change as well. In the interest to get a one-storage element with unity transfer factor we have to insert a member with gain $K_{p}$ between the integrator of PF controller and reference signal of the inner loop. The one-storage reference model with time constant $T_{m}$ gets the sum of the input signal of above member $\left(\omega_{r}\right)$ and the signal $\omega_{t m}$ compensating the load effect for the model. So the dynamics of the reference model can be described by the following differential equation:

$$
\dot{\omega}_{m} \mathrm{~T}_{\mathrm{m}}+\omega_{m}=\omega_{r}+\omega_{t m}
$$

in which the index $m$ refers to the model.

If we divide Eq. (3) with $T_{m}$ and apply the designation $q_{m}=1 / T_{m}$, we get the following equation:

$$
\dot{\omega}_{m}+\mathrm{q}_{\mathrm{m}} \omega_{m}=\mathrm{q}_{\mathrm{m}}\left(\omega_{r}+\omega_{t m}\right)
$$

The differential equation of the one-storage controlled plant is as follows:

$$
\dot{\omega}+\left(K_{p} A_{i}\right) \omega=\left(K_{p} A_{i}\right) \omega_{r}-A_{i} i_{t} .
$$

The factor $K_{p}$ can be described as the sum of $\mathrm{K}_{\mathrm{p} 0}$ determined on the mean $A_{i}$ and $\Delta K_{p}$ accomplished by the adaptation algorithm. So thus:

$$
K_{p} A_{i}=\left(\mathrm{K}_{\mathrm{p} 0}+\Delta K_{p}\right) A_{i}=\mathrm{q}+\Delta q,
$$

where $\mathrm{K}_{\mathrm{P} 0}$, and $\mathrm{q}$ are constant.

In this case we assume that the change of $A_{i}$ is slow from the viewpoint of adaptation, therefore the effect of this change can be neglected.

Substituting Eq. (6) into Eq. (5) we get:

$$
\dot{\omega}+(\mathrm{q}+\Delta q) \omega=(\mathrm{q}+\Delta q) \omega_{r}-A_{i} i_{t} .
$$

By using Eq. (4) and (7) and substituting the expression of model error $\varepsilon=\omega_{m}-\omega$ the dynamic equation will be: 


$$
\dot{\varepsilon}=-\mathrm{q}_{\mathrm{m}} \varepsilon+x \omega-x \omega_{r}+\mathrm{q}_{\mathrm{m}} \omega_{t m}+A_{i} i_{t},
$$

where $x=(\mathrm{q}+\Delta q)-\mathrm{q}_{\mathrm{m}}$.

For the sake of that the system should follow the model, the error of dynamics is stable asymptotically. In the interest of determination $\Delta q$ the following Lyapunov function should be composed:

$$
V=\frac{1}{2}\left(\varepsilon^{2}+\beta x^{2}\right)
$$

where $\beta$ is a positive number.

At choosing the Lyapunov function both purposes, i.e. the termination of the model error and loop gain deviation have been taken account.

The time derivative of the Lyapunov function is:

$$
\dot{V}=\varepsilon \dot{\varepsilon}+\beta x \dot{x}
$$

Substituting Eq. (8) into Eq. (10) the following equation is valid:

$$
\dot{V}=-\mathrm{q}_{\mathrm{m}} \varepsilon^{2}+\varepsilon x \omega-\varepsilon x \omega_{r}+\varepsilon\left(\mathrm{q}_{\mathrm{m}} \omega_{t m}+A_{i} i_{t}\right)+\beta x \dot{x} .
$$

If

$$
\varepsilon \times \omega-\varepsilon \times \omega_{r}+\beta \times \dot{x}=0,
$$

that is

$$
\dot{x}=\varepsilon\left(\omega_{r}-\omega\right) / \beta
$$

and

$$
\varepsilon\left(\mathrm{q}_{\mathrm{m}} \omega_{t m}+A_{i} i_{t}\right)<0
$$

then

$$
\dot{V}<-\mathrm{q}_{\mathrm{m}} \varepsilon^{2}
$$

The above equation is a negative definite function that shows the asymptotic stability of the error dynamic Eq. (8). By using the relations (6), (8) and (12) the following adaptation algorithm is true:

$$
\Delta \dot{K}_{p}=\gamma \varepsilon\left(\omega_{r}-\omega\right),
$$

where $\gamma$ may be an arbitrary positive number.

The inequality (13) shows how we have to change the signal $\omega_{t m}$ representing the load of model.

If

$$
\varepsilon>0, \text { then } \omega_{\mathrm{tm}}<-\frac{\left|i_{t}\right|_{\max } A_{i}}{\mathrm{q}_{\mathrm{m}}},
$$

respectively if

$$
\varepsilon<0, \text { then } \omega_{\mathrm{tm}}>\frac{\left|i_{t}\right|_{\max } A_{i}}{\mathrm{q}_{\mathrm{m}}} .
$$




\section{Convergence test}

To analyze the convergence of the parameter adaptive speed control a square reference signal with $0.2 \mathrm{sec}$ period time has been applied. Figures 3. - 5. show changes of $K_{p}$ gaining factor during the adaptation process. The value of adaptation coefficient ( $\Gamma$, which is the $\gamma$, modified according to sampling time) and therefore convergence speed are the lowest in Fig. 3. $(\Gamma=0,00001)$. It is clear in the upper figure that with low initial value $K_{p}$ practically reaches its steady-state value in 8 seconds and the pulsation is low. With initial value higher than the steady state value of $K_{p}$, convergence is slower, more 8-second long simulation cycle is needed to reach the steady-state.

In Figure 4. with one order of magnitude higher $\Gamma$ value has been set than in the previous case. So adaptation process has speeded up significantly. At the same time the pulsation of steady-state $K_{p}$ value has become significant, its value oscillates between 1 and 1.5. With another order of magnitude increasing in adaptation factor (Fig. 5.) adaptation process speeds up away but the pulsation in the gaining factor is so high (characteristically between 1 and 3, sometimes it fluctuates even more) that in practical cases it is not acceptable.

\section{Summary for the simulation results of model reference parameter adaptive control}

According to the simulation investigations convergence of the model reference parameter adaptive PF control at switched reluctance drives with significant torque ripples can be ensured with the next conditions:

- Input of the first-order reference model only determines its output when the filtered current reference signal is lower with a given $\Delta \mathrm{I}$ value (in the simulation it has been set to $1 \mathrm{~A}$ ) than its limit otherwise output is identical to the speed feedback signal. So the model works like a firstorder proportional lag element only in operation without current limitations.

- Adaptation is executed when two conditions are true at the same time:

- Filtered current reference signal is lower with a given $\Delta \mathrm{I}$ value (in the simulation it has been set to $1 \mathrm{~A}$ ) than current limit and

- Absolute value of the speed error signal is higher than a given $\Delta \mathrm{n}$ value (in the simulation it has been set to 20 revolution $/ \mathrm{min}$ ).

- Setting the initial value of the $K_{p}$ gain factor to low is practical. In this case convergence is faster and safer.

- The appropriate selection of $\Gamma$ adaptation factor also has important effect on the sufficient convergence: the faster the adaptation, the higher the variation of the adaptation factor in steady state.

Because of the restrictions described in point 2. adaptation practically works only in a relatively narrow speed error track (adaptation range) which is equal to approximately 20-100 revolution/min absolute value of speed error. The drawback of this limitation is the relatively short time for the algorithm to operate. But the convergence of the algorithm is extremely fast which significantly reduces the effect of this drawback. Two more important advantages emerge when adaptation works only with small speed errors. First of all the controller at changing drive parameters adapts to parameters around the value specified by speed reference signal which also assists to speed the adaptation. The other significant positive effect is the disappearing of the problem coming from nonlinear systems that the response of the system can even differ in its character when the value, amplitude of the reference signal is changed.

Requirement described in point 3 . is easily provable. In the deduction of the adaptation algorithm time constant of the closed current control loop was neglected. The effect of this time constant causes high frequency oscillations at high loop gains which makes the convergence difficult what's more impossible. 

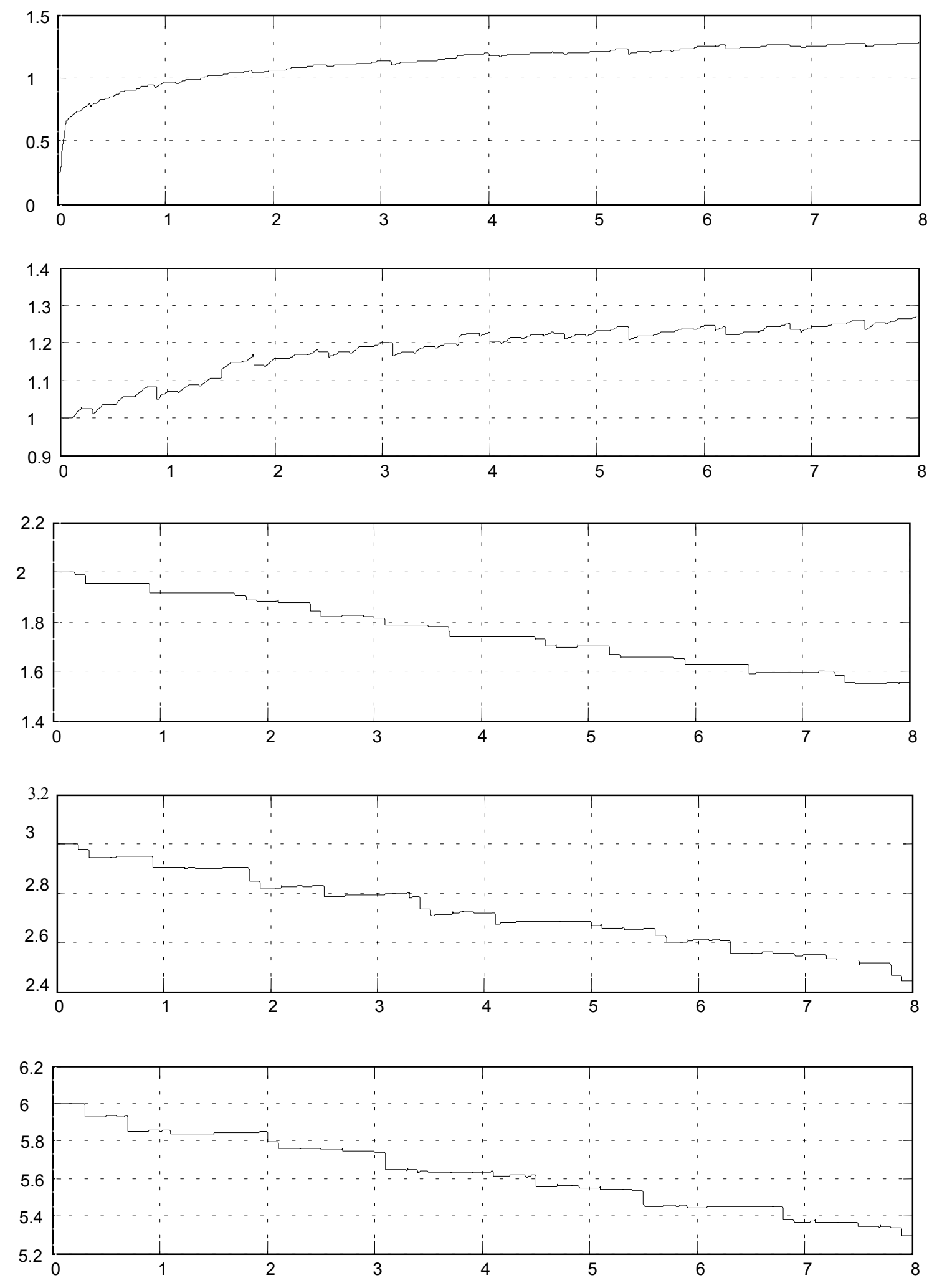

Fig. 3: The convergence of $K_{p}$ value at $\Gamma=0,00001$ 


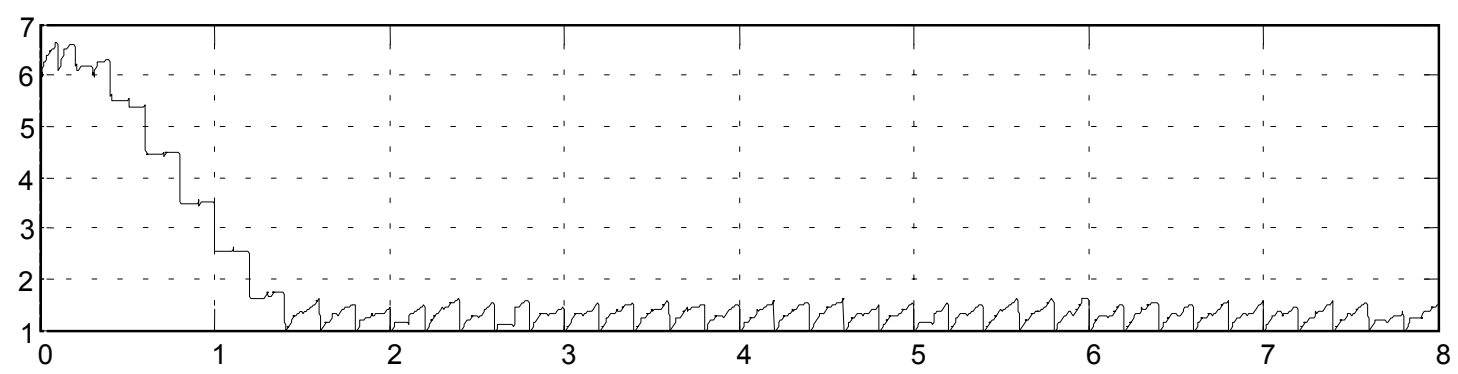

Fig. 4: The convergence of $K_{p}$ value at $\Gamma=0,0001$

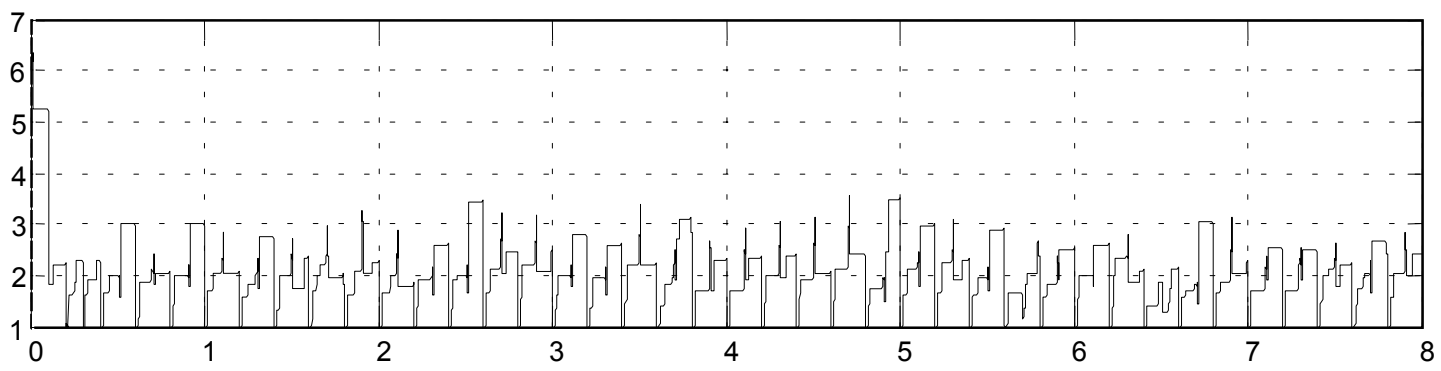

Fig. 5: The convergence of $K_{p}$ value at $\Gamma=0,001$

Although the too low initial value of gain effects on the initial quality of the speed control (because on the effect of the integrator of the PF controller lower frequency oscillations can emerge) but it is outside of the control loop (this oscillation also appears on the input of the first-order lag element) so it takes less effect on the convergence of the adaptation.

\section{Results}

In Fig. 6 and Fig. 7 two of many executed simulations are shown. Fig. 6 shows the run-up with speed controller of PF-type (an integral element with Proportional Feedback, $\Delta K_{p}=0$ ), while Fig. 7 with model reference parameter adaptive control (Eq. 15, Eq. 16) and in both cases with turn-on and turn-off angles depending on the speed and current reference and with current reference compensation (Eq. 1).

The tests were completed by the described drive system. The test results have supported our theoretical investigations. The loading machine was a DC motor. Its inertia is about a triple of that of SRM. Fig. 8. shows the speed and current curves in the course of starting without current reference compensation. The upper curve is the speed $(1500 \mathrm{rpm})$, the lower one is the current flowing in the common point of stator windings $(10 \mathrm{~A} / \mathrm{div})$. It is related to the no-load operation mode.

\section{Conclusion}

Basic structure of the speed controller is PF type which firstly provides overshootless with its structure. On the other hand it ensures the fast compensation of speed variation caused by jump in the motor load. I have applied parameter adaptive control in the inner proportional feedback loop of the PF controller. Thus I have managed to provide adaptation closest to the changing parameter and at the same time the most simplest but effective algorithm has been deducted. Primarily its significance is not the implementation of the deducted controller (real time computation demand) but because of tuning a multi-parameter control is significantly more difficult compared to the proposed single-parameter structure. Thanks to the first-order model adaptation algorithm does not need angular acceleration measuring. The deducted algorithm is extremely fast and it can provide stability even at jumps in the load. Only the factor changes jump-like 

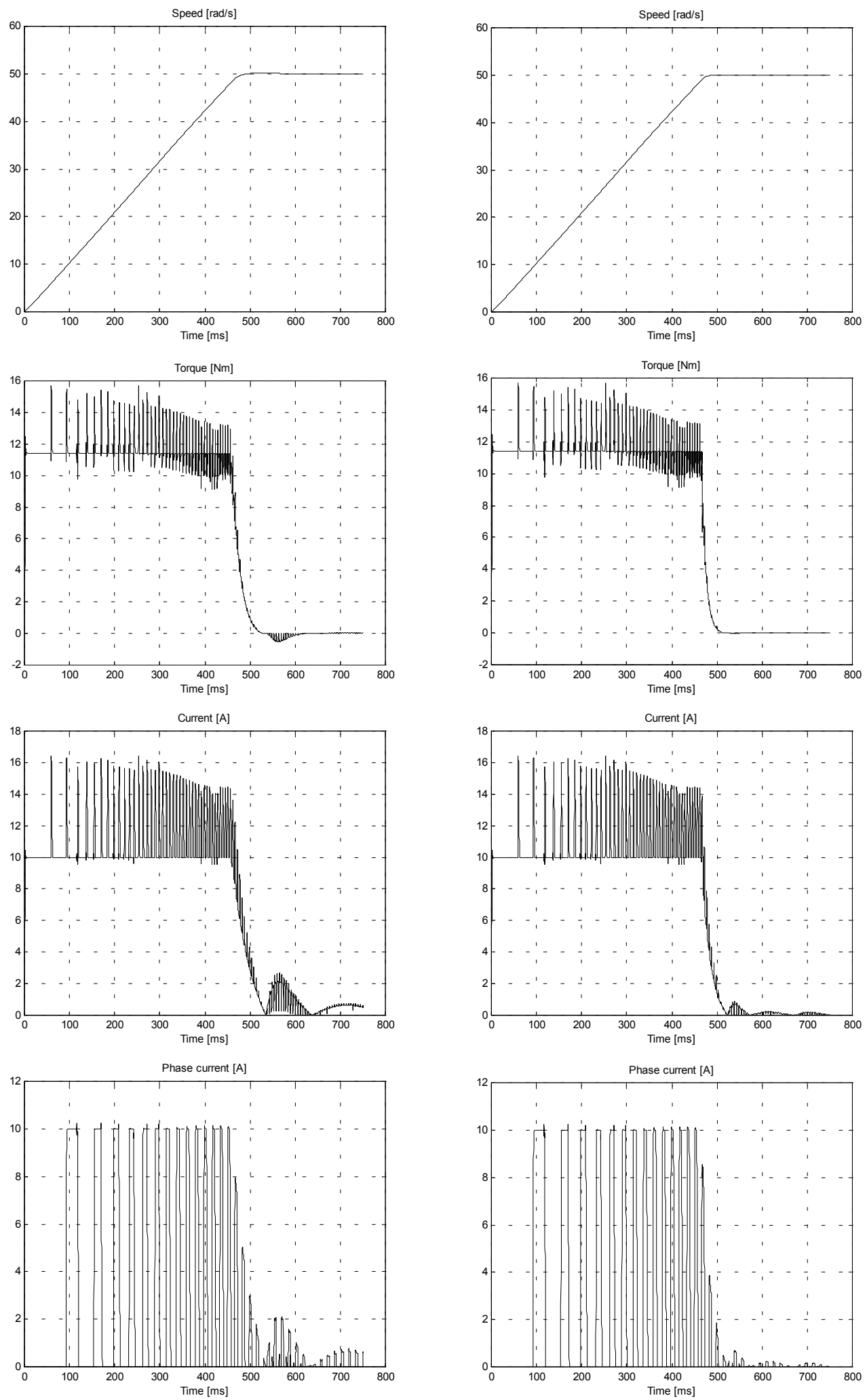

Fig.6: Simulation results with PF-type speed control $\left(\Delta K_{p}=0\right)$

Fig.7: Simulation results with model reference parameter adaptive speed control 


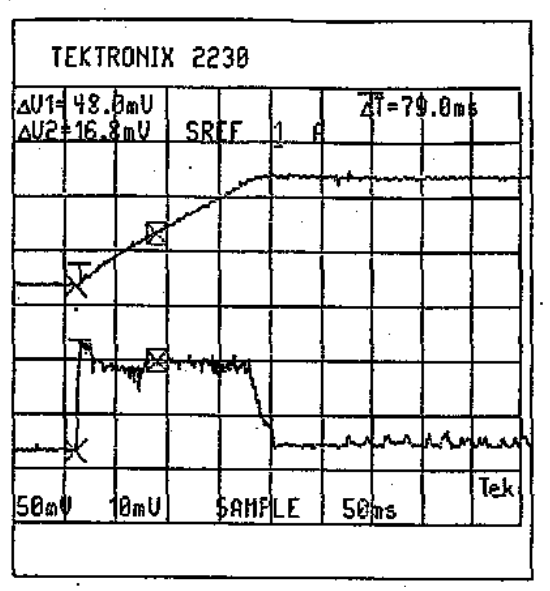

Fig. 8: Oscillograms of the speed and current, model reference parameter adaptive control

which takes into consideration the load for the model and speed error caused by jump in the load is compensated by the slower changing P and constant I element of the controller. With appropriate choice of the adaptation range convergence of the adaptation can even be ensured at SRM drives with significant torque ripples. Although normally because of the nonlinear characteristic of the adaptation, response of the system also depends on value of the jump in the reference signal this does not cause any problems in the proposed adaptive control as adaptation only happens in a narrow track of the reference signal named adaptation range. The wideness of the adaptation range is determined firstly by the torque ripples (with very low error adaptation does not work) and on the other hand the section where adaptation algorithm can work that is where limitations does not take effect depends on the agility of the speed controller (constituting time constant). At servo-drives speed error belongs to it is about 50-100 revolution/min so it could be seen approximately independent from the jump magnitude in the reference signal. The experiences show that the model reference parameter adaptive control suggested in this paper works without overshooting. Though this method requires a longer calculation period it is less sensible to the variations of parameters. The simulations and experimental results demonstrate that the proposed method is a promising tool to control the SRM drives.

\section{References}

[1]. Miller, T.J.E. Brushless permanent-magnet reluctance motor drives. Oxford University Press. 1989

[2]. Diep, M.V. - Szamel, L.: Up-to-date Control Strategy in the Regulators of Robot Drives. PEMC'90, Budapest. 1990. pp. $811-815$

[3]. Bose, B.K. - Miller, T.G.E. Microcomputer Control of Switched Reluctance Motor. IEEE/IAS Annual Meeting. 1985. pp. 542-547.

[4]. Borka, Jozsef. - Szamel, Laszlo. Modern Strategy for Controlling Robot Drives. Conference Automation'92, Budapest. 1992. pp. 392-401

[5]. Stankovic, Alexander. - Tadmor, Gilead. On Torque Ripple Reduction in Current-Fed Switched Reluctance

Motors. IEEE Transactions on Industrial Electronics, Vol. 46, No. 1, February 1999. pp. 177-183

[6]. Szamel, Laszlo. Ripple reduced control of switched reluctance motor drives, EDPE2001, International

Conference on Electrical Drives and Power Electronics, Podbanské (Slovak Republik), 3-5 October 2001. pp. 48-53

[7]. Szamel, Laszlo. Adaptive Ripple Reduced Control of SRM Drives, SPEEDAM2002, Symposium on Power

Electronics, Electrical Drives, Automation \& Motion, Ravello (Italy), 11-14 June 2002. pp. B4/19-B4/23

[8]. Szamel, Laszlo. Model Reference Adaptive Control of SRM Drives, EPE-PEMC 2002, $10^{\text {th }}$ Int. Power

Electronics and Motion Control Conf., Cavtat \& Dubrovnik (Croatia), 9-11 September 2002. pp. 466, CD-ROM:

Paper No. T11-007

[9] Szamel, Laszlo. Investigation of Model Reference Parameter Adaptive SRM Drives, EPE-PEMC 2004, $11^{\text {th }}$ Int. Power Electronics and Motion Control Conf., Riga (Latvia), 2-4 September 2004, Full paper A95117 on CD_ROM 